\title{
Proteomic-Test als Ergänzung für Zystoskopie
}

Blasenkarzinom-Patienten müssen aufgrund hoher Rezidivraten lebenslang regelmäßig nachuntersucht werden. Wie eine Übersichtsarbeit zu nicht-invasiven Methoden für die Nachuntersuchung von Blasenkarzinom-Patienten ergab, kann bislang kein Proteomic-Test die Zystoskopie ersetzen (Gaston KE, Grossman B, 2010, Methods Mol Biol 641:303-323). Diese Tests ermöglichen es jedoch ergänzend, visuell nicht sichtbare Läsionen zu detektieren. Dabei ist NMP22 nach Einschätzung der Autoren der derzeit beste verfügbare Proteomic-Test zum Nachweis von Blasenkrebs.

Proteomic-Tests sind eine nicht-invasive Diagnosetechnik, mit der urinlösliche tumorspezifische Marker nachgewiesen werden. Das nukleäre Matrixprotein 22 (NMP22) ist einer dieser Marker.

NMP22 ist der einzige von der US-amerikanischen Food and Drug Administration (FDA) sowohl für das Screening als auch das Monitoring zugelassene Proteomic-Test. Im Monitoring von Patienten nach Blasenkrebs erreicht der NMP22-Point-of-Care-Test eine Sensitivität von $49,5 \%$ und eine Spezifität von $87,3 \%$. Blasenkarzinome lassen sich mit NMP22 vor allem in Anfangsstadien mit deutlich höherer
Sensitivität nachweisen als mit der zytologischen Untersuchung des Urins.

Wichtig bei der Durchführung eines NMP22Tests ist die Beachtung von Ausschlusskriterien (z.B. Harnwegsinfekte, Katheter und Nephrolithiasis). Studien mit 212 Patienten (NMP22 ${ }^{\circledR}$ BladderChek $^{\circledast}$ ) bzw. 608 Patienten (NMP22 ${ }^{\circledR}$ Elisa) - jeweils mit Verdacht auf Blasenkrebs -

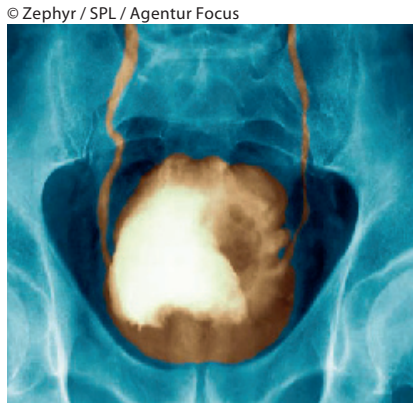

Blasenkarzinom - gefärbte Röntgenaufnahme ergaben unter Einhaltung von definierten Ausschlusskriterien eine Sensitivität von $82 \%$ bzw. $88 \%$ und eine Spezifität von 98\% bzw. 99\% - unabhängig von der Stadieneinteilung (Oehr P, Schroeder A, 2006, TumorDiagn u Ther 27: 205-210; Ponsky LE et al., 2001, J Urology 166: 75-78). Durch Kombination von zytologischer Untersuchung und Proteomic-Test kann die Sensitivität deutlich gesteigert werden, wie die Ergebnisse einer aktuellen Studie zeigen (Schwentner C et al., 2010, AUA: \#1171): Die Kombination von Zytologie plus NMP22-Test (NMP22 ${ }^{\circledR}$ Elisa) erreichte eine Sensitivität von $90,4 \%$, die Sensitivität der Zytologie allein betrug $80,8 \%$.

Quelle: Informationen der Matritech $\mathrm{GmbH} /$ Alere $\mathrm{GmbH}$

\section{Lungenkarzinom}

\section{COPD limitiert die Tumortherapie}

Chronic Obstructive Pulmonary Disease (COPD) und Lungenkarzinom sind eng assoziiert. Dabei ist die COPD per se ein starker Prädiktor für die Karzinomentwicklung. Die COPD schränkt zudem die Behandlungsmöglichkeiten der Tumorerkrankung ein.

Ein chirurgischer Eingriff vermindert beim Lungenkarzinom die ohnehin reduzierte Atmungsreserve des COPD-Patienten, so Joachim Lorenz, Lüdenscheid. Risikofaktoren für postoperative respiratorische Probleme sind Alter, COPD und niedriges postoperatives forciertes Ein-SekundenAusatemvolumen (FEV1).

COPD-Patienten mit nicht resektablem Lungenkarzinom sind häufig auch einer pulmonalen Radiotherapie nicht zugänglich. „Der Flurschaden bei perkutaner Strahlentherapie ist qualitativ etwa so groß wie bei einem operativen Eingriff", so Lorenz. Allerdings könne es mit modernen Bestrahlungsplanungsmethoden wie der atmungsgetriggerten Radiotherapie, gelingen, auch Patienten mit schlechter pulmonaler Funktion einer Bestrahlung zu unterziehen, möglicherweise sogar in kurativer Dosierung.
Bei der Chemotherapie müsse die pulmonale Toxizität mancher Zytostatika mit in die Planung einbezogen werden.

\section{Zielgerichtete Therapien in der Pipeline} Große Erwartungen haben Experten an neue zielgerichtete Therapien. In der klinischen Entwicklung für die Indikation nichtkleinzelliges Lungenkarzinom (NSCLC) sind derzeit u.a. der Tyrosinkinaseinhibitor Afatinib und der Angiokinaseinhibitor BIBF 1120. BIBF 1120 hemmt die drei maßgeblich an der Neubildung von Blutgefäßen beteiligten Wachstumsfaktorrezeptoren: VEGFR (Vascular Endothelial Growth Factor Receptor), PDGFR (PlateletDerived Growth Factor Receptor) und FGFR (Fibroblast Growth Factor Receptor), berichtete Rainer Wiewrodt, Münster. $D J B$ Quelle: Veranstaltung der Boehringer Ingelheim Pharma $\mathrm{GmbH} \&$ Co. KG

\section{Doppelt refraktäre CLL: gutes Ansprechen auf Ofatumumab}

Erste Ergebnisse der Endauswertung der zentralen Zulassungsstudie zu Ofatumumab (Arzerra ${ }^{\circledR}$, GlaxoSmithKline $\mathrm{GmbH} \&$ Co. KG) bestätigen die in der Zwischenauswertung gezeigten Daten zur Sicherheit und Verträglichkeit einer Ofatumumab-Monotherapie bei Patienten mit chronischer lymphatischer Leukämie (CLL), die auf Fludarabin und Alemtuzumab refraktär waren (Wierda W et al., 2010, J Clin Oncol 28: 1749-1755). In der multizentrischen Zulassungsstudie erhielten 95 CLL-Patienten, die nicht auf Fludarabin und Alemtuzumab angesprochen hatten, Ofatumumab als Monotherapie. Die objektive Ansprechrate (ORR) - ermittelt von einem „Independent Review Committee" - betrug $51 \%$. In der Studie waren darüber hinaus 128 Fludarabin-refraktäre CLL-Patienten eingeschlossen, die aufgrund einer ausgeprägten Lymphadenopathie („bulky disease") nicht für eine Therapie mit Alemtuzumab geeignet waren. 